\title{
Unlike Particle Correlations and the Strange Quark Matter Distillation Process
}

\author{
D. Ardouin*, Sven Soff, C. Spieles, S. A. Bass, H. Stöcker \\ Institut für Theoretische Physik, J. W. Goethe-Universität, \\ Postfach 1119 32, D-60054 Frankfurt am Main, Germany; ${ }^{\dagger}$ \\ D. Gourio, S. Schramm \\ GSI Darmstadt, Postfach 1105 52, D-64220 Darmstadt, Germany; \\ C. Greiner \\ Institut für Theoretische Physik, J. Liebig-Universität, \\ Heinrich-Buff-Ring 16, D-35392 Gießen, Germany; \\ R. Lednicky \\ Institute of Physics of the Academy of Sciences of the Czech Republic, \\ Na Slovance 2, 18040 Prague 8, Czech Republic; \\ V. L. Lyuboshitz ${ }^{\S}$ \\ JINR Dubna, 141980, Moscow, Russia; \\ J.-P. Coffin, C. Kuhn \\ CRN Strasbourg, Université L. Pasteur, Strasbourg, France.
}

We present a new technique for observing the strange quark matter distillation process based on unlike particle correlations. A simulation is presented based on the scenario of a two-phase thermodynamical evolution model.

\footnotetext{
*on leave from University of Nantes, U.M.R. Subatech

${ }^{\dagger}$ supported by GSI, BMBF, DFG and Buchmann Fellowship

${ }^{\ddagger}$ supported by GA AV CR, Grant No. A1010601 and GA CR, Grant No. 202/98/1283

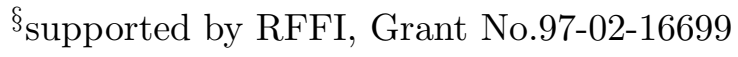




\section{MOTIVATION}

The possibility to create strangelets or droplets of metastable cold strange quark matter in ultra-relativistic collisions has been proposed and studied by several authors [1]. The existence of such exotic states, as well as metastable exotic multi-strange baryonic objects (MEMO's), has fundamental importance in cosmological models and in the underlying description of strong interactions. Several experiments are being carried out at the Brookhaven AGS (E864, E878) and the CERN SPS (NA52), which look for strangelet production (searching for small $Z / A$ ratios) [8-10].

Among the different theoretical approaches [1] [0], a mechanism of separation of strangeness from anti-strangeness (distillation process) has been proposed [4] during hadronization of a system at finite baryon densities. This scenario, which assumes a first order phase transition, predicts a relative time delay between the production of strange and anti-strange particles. In the present paper, we propose to search for this delay with the help of the novel correlation method proposed in [11, [12]. It exploits the sensitivity of the directional dependence of the correlation function of two non-identical particles to the time order of their emission. This new technique can allow for the study of the transient strange quark matter state even if it decays on strong interaction time scales [13 15].

A different method, exploiting $K_{s}^{0} K_{s}^{0}$ correlations, also allowing for a study of the relative delays (but not their sign) in $K$ and $\bar{K}$ emission was proposed in 16. It is based on the fact that the $K_{s}^{0} K_{s}^{0}$ system, due to the positive CP parity of $K_{s}^{0}$, triggers out the symmetric combination of the $K^{0} \bar{K}^{0}$ states, thus leading to the familiar Bose-Einstein correlation pattern [17]. This pattern, contrary to the case of non interacting identical bosons, is however substantially modified by the effect of the strong final state interactions [18].

Here we will use the general method of [11,12] to study the impact of the time delays on the correlations in $K^{+} K^{-}$pairs. The choice of this system, similar to the case of neutral kaons, apart from the possibility to determine the signs of the time delays, has the advantage of weaker distortions caused by the resonance production (more than $50 \%$ of kaons are pre- 
dicted to originate from direct emission [19]), as compared to pions, while the experimental feasibility is better than in the case of neutral kaons.

\section{THE MIXED PHASE THERMODYNAMICAL APPROACH}

The dynamical evolution of the mixed phase consisting of a quark gluon plasma and hadronic gas will be described in a two-phase model which takes into account equilibrium as well as non-equilibrium features [20,21].

Within this model, two main assumptions are made. Firstly, the QGP is surrounded by a layer of hadron gas and equilibrium ( described by Gibbs conditions) is maintained during the evolution. Secondly, non-equilibrium evaporation is incorporated by a time dependent emission of hadrons from the surface of the hadronic fireball. Within this model, it is possible to follow the evolution of mass, entropy and strangeness fraction $f_{s}$ of the system and extract relative yields of particles which compare reasonably well with experimental data [19].

One specific feature of these calculations is the prediction that the system quickly enters the strangeness sector leaving the usual $\mu_{\mathrm{q}}-T$ plane of the phase diagram. While the net-baryon number $A_{B}$ decreases during the hadronization process, the s quark chemical potential $\mu_{\mathrm{s}}$ increases from $\mu_{\mathrm{s}}=0 \mathrm{MeV}$ (for $f_{s}^{\text {ini }}=0$ if we start with a pure $\mathrm{u}, \mathrm{d}$ quark

phase) to several tens of MeV. Consequently, the strangeness fraction $f_{s}=\left(N_{s}-N_{\bar{s}}\right) / A_{\text {tot }}$ increases. This is the so-called "distillation process" [4 which can result in the formation of stable or metastable blobs of strange matter in the case of low bag model constants $B^{1 / 4}<180 \mathrm{MeV}$. (In this case, a cooling of the system is predicted.) Strange and antistrange quarks, produced in equal amount in the hot plasma, do not hadronize at the same time. Because of the excess of massless ( $u$ and $d$ ) quarks (as compared to their anti-quarks) in the case of a baryon rich plasma, the hadronization of $K^{+}$and $K^{0}$ (containing $\bar{s}$ quarks) will be favored preferentially to $K^{-}$and $\bar{K}_{0}$ ( containing s quarks). The evaporation from the surface of the hadron gas, which is rich of anti-strangeness, carries away $K^{+}, K^{0}, \ldots$ thus charging up the remaining mixed system with net positive strangeness. Fig.1 shows, 
for two rather different bag constants, the time evolution [19] of the baryon number $A$, the strangeness fraction $f_{s}$ and the temperature $T$ for initial entropy $S / A_{\text {ini }}=10,45$ and $f_{s}=0$. Another prediction of interest is the hadronic freeze-out time. Strange and antistrange hadrons are not emitted at the same time since the hadron densities in the outer layer are dictated by the strongly time dependent chemical potentials and the time dependent temperature 20,19]. One should also notice that the difference between $K^{+} N$ and $K^{-} N$ cross sections in a baryon rich gas will slow down the diffusion of negative strangeness (s), thus leading to an additional creation of time differences [20,19].

In the present work, this time separation characterizes the transient existence of the plasma. In the following, we will show that it can be used to search for the distillation process by using correlation techniques of unlike particle pairs [22,23,18].

\section{UNEQUAL PARTICLE CORRELATIONS: TIME-ORDERING SENSITIVITY}

Particle correlations at small relative momentum are mainly driven by strong and Coulomb final state interactions (in the case of unlike particle pairs).

At high bombarding energies, this effect has been often treated with a well known sizeindependent Coulomb correction factor. Contrary, at low energies, the Coulomb effect becomes the main tool for the study of this evolution due to larger time sequence of emission.

Unlike particle correlations have been used experimentally and theoretically for more than 15 years [18,22, particularly for the study of heavy ion collision mechanisms around the Fermi energy 22 25]. Particularly, evidence for three-body Coulomb effects on twoparticle correlations was observed in 24] and taken into account to describe a variety of two-particle correlation patterns using classical trajectory calculations 250 or a complete three-body quantum approach [11,26] in the adiabatic limit. The sensitivity of unlike particle correlations to the order of particle emission was pointed out and studied using both quantum [11,12] and classical trajectory [27] approaches. In the framework of the former one and under

the assumption of sufficiently small density in phase space, the two-particle correlation 
function at a given relative c.m.s. momentum $\vec{q}=2 \vec{k}$ is determined by the modulus squared of the two-particle amplitude averaged over the relative c.m.s. coordinates $\vec{r}^{*}$ of the emission points. The sensitivity of the correlation function to the time delays, or generally to the space-time asymmetries in particle production, appears due to the dependence of the twoparticle amplitude on the scalar product $\vec{k} \vec{r}^{*}$. In particular, in the limit of large relative emission times $t=t_{1}-t_{2}, v|t| \gg r$, the Lorentz transformation from the source rest frame to the two-particle c.m.s ( $v$ and $\gamma$ are the pair velocity and Lorentz factor):

$$
r_{L}^{*}=\gamma\left(r_{L}-v t\right), \quad r_{T}^{*}=r_{T}
$$

indicates that the vector $\vec{r}^{*} \approx-\gamma \vec{v} t$ is only slightly modified by averaging over the spatial location of the emission points in the rest frame of the source. So the vector $\vec{r}^{*}$ is nearly parallel or antiparallel to the pair velocity $\vec{v}$, depending on the sign of the time difference $t$. The dependence of the amplitude on $\vec{k} \cdot \vec{r}^{*}$ is thus transformed into the dependence of the correlation function on $-\vec{k} \cdot \vec{v} \gamma t$. Therefore, the sensitivity to the sign of the time difference $t$ is due to the odd part, in $\vec{k} \cdot \vec{v}$, of the correlation function. The mean relative emission time, including its sign, can be determined by comparing the correlation functions $R^{+}$and $R^{-}$corresponding to $\vec{k} \cdot \vec{v}>0$ and $<0$ [12]. Noting that for particles of equal masses the sign of $\vec{k} \cdot \vec{v}$ coincides with the sign of the velocity difference $v_{1}-v_{2}$, we can see the simple classical meaning of the above selection. It corresponds to the intuitive expectation of different particle interaction in the case when the faster particle is emitted earlier as compared to the case of its later emission.

For charged particles characterized by a large Bohr radius, $|a| \gg\left\langle r^{*}\right\rangle,|\operatorname{Re} f|(f$ is the amplitude due to the strong interaction), the ratio $\left(1+R^{+}\right) /\left(1+R^{-}\right)$at very small $q$ takes on a simple analytical form [28]:

$$
\left(1+R^{+}\right) /\left(1+R^{-}\right) \approx 1+2\left\langle r_{L}^{*}\right\rangle / a \rightarrow 1-2\langle\gamma v t\rangle / a,
$$

where the arrow indicates the limit $v|t| \gg r$. Thus, for $K^{+} K^{-}$-system $(a=-111 \mathrm{fm})$, each fm in the asymmetry $\left\langle r_{L}^{*}\right\rangle$ or $\langle\gamma v t\rangle$ transforms in a $2 \%$ change of the $\left(1+R^{+}\right) /\left(1+R^{-}\right)$ratio 
at $q \rightarrow 0$. The correlation functions $R^{+}$and $R^{-}$as predicted in [11,12] well agree with the proton-deuteron correlation functions measured in low energy nuclear collisions studied at GANIL [29, 14, 30, 31]. As proposed in [12], this technique can be extended for a study of the emission time differences between any kinds of interacting unlike particles.

In preliminary studies using simple event generators, it has been demonstrated [13] [15] that $K^{+} K^{-}$pairs with mean emission time differences as small as $\pm 5 \mathrm{fm} / \mathrm{c}$ can give rise to observable differences between $R^{+}$and $R^{-}$correlation functions. It has been also found that most of the effect at $q<10 \mathrm{MeV} / \mathrm{c}$ originates from the Coulomb interaction between the two kaons and that, in full correspondence with Eq. (2), the later (on the average) emission of $K^{-}$'s is associated with the $\left(1+R^{+}\right) /\left(1+R^{-}\right)$ratio less than unity.

In this paper, we will use this method in conjunction with the dynamical two phase description (see previous section) in order to quantitatively demonstrate its sensitivity to the predicted delays between the $K^{+}$and $K^{-}$emission related to the production of a transient strange quark matter state.

Three sets of parameters [15,19] have been chosen: set $1 \& 2$ (representing $\mathrm{Au}+\mathrm{Au}$ at AGS energies [19]):

initial mass $A=394$, entropy per baryon $S / A=10$, initial net strangeness $f_{s}=0$; set 1: $B^{1 / 4}=160 \mathrm{MeV}$, strangelets (albeit metastable) are formed [19]. set 2: $B^{1 / 4}=235 \mathrm{MeV}$, strangelets are not distilled [19]. They are not (meta-)stable. set 3 ( representing $\mathrm{S}+\mathrm{Au}$ at SPS energies [19): bag model constant $B^{1 / 4}=235 \mathrm{MeV}$, initial mass $A=100$, entropy per baryon $S / A=45$, initial net strangeness $f_{s}=0$.

In the case of the low bag constant $\left(B^{1 / 4}=160 \mathrm{MeV}\right)$ - set 1 - a cooling of the system is predicted leading to rather long kaon emission times. In the early stage mainly $K^{+}$'s are emitted (see Fig. 1, l.h.s. column) so that a cold strangelet emerges in a few tens fm/c. According to Eqs. (1), (2) and the corresponding discussion, the later emission of $K^{- \text {'s }}$ should lead to the correlation function ratio $\left(1+R^{+}\right) /\left(1+R^{-}\right)$less than unity provided 
that the asymmetry $\left\langle r_{L}^{*}\right\rangle$ in $K^{+} K^{-}$c.m.s. is dominated by the time asymmetry term $\langle v t\rangle$. As seen from table 1 and Fig. 2 this is indeed the case: the spatial asymmetry term $\left\langle r_{L}\right\rangle$ contributes by less than $10 \%$ (in the same direction); the predicted value of the deviation of the correlation function ratio from unity of $-26 \%$ at $q \rightarrow 0$ is in good agreement with the intercept value of the calculated ratio curve with the ordinate axis. Also, the deviation from unity remains important and statistically meaningful up to the region $q \approx 30 \mathrm{MeV} / \mathrm{c}$. Thus, in spite of experimental difficulties to measure correlations down to a few $\mathrm{MeV} / \mathrm{c}$, the signal proposed for consideration is accessible to usual correlation measurements. Since for various two-particle systems produced in the mid-rapidity region, the ordinary dynamical mechanisms lead to the intercept values deviating from unity by less than $10 \%$ [28], we can consider the eventual observation of a large negative asymmetry in the $K^{+} K^{-}$correlation function ratio as a signal of the strangelet formation.

In the case of the high bag constant $\left(B^{1 / 4}=235 \mathrm{MeV}\right)$ - set 2 and 3 - the system heats up slightly. This results in a fast hadronization [20] and, subsequently, in a small difference between the $K^{+}$and $K^{-}$emission times [19] (see the middle and r.h.s. columns in Fig. 1). Similar to the previous case the $K^{+}$'s are emitted earlier than $K^{-}$'s. Also the asymmetry $\left\langle r_{L}^{*}\right\rangle$ is dominated by the time asymmetry term $\langle v t\rangle$, the spatial one, $\left\langle r_{L}\right\rangle$, contributing by about $25 \%$ in the same direction (see table 1, rows 2 and 3 ). However, the asymmetry effect is now much weaker (Fig.2): the predicted intercepts with the ordinate axis of the correlation function ratio deviate from unity by $-4.7 \%$ and $-2.3 \%$ for sets 2 and 3, respectively.

Clearly, in this case, such a relatively weak asymmetry effect cannot be considered as a signal of the strangeness distillation without detailed studies of the impact of the ordinary mechanisms (rescattering effects, for example).

Regarding the width of the effect seen in the correlation function ratios (Fig. 2), it decreases with the increasing $\left\langle r^{*}\right\rangle$ (see table 1) in correspondence with the narrowing of the correlation effect itself. Thus, for set $1\left(\left\langle r^{*}\right\rangle \approx 18 \mathrm{fm}\right)$ the effect rapidly vanishes with increasing $q$ and extends up to about $30 \mathrm{MeV} / \mathrm{c}$ only. For sets 2 and $3\left(\left\langle r^{*}\right\rangle \approx 6 \mathrm{fm}\right.$ in both cases) the deviation from unity is softer in the same range of $q$ values and extends up to 
about $60 \mathrm{MeV} / \mathrm{c}$.

\section{CONCLUSIONS}

We have presented a novel method which can be applied to characterize the possible existence of a strange quark matter distillation process in ultra-relativistic heavy-ion collisions. The method is based on the predictions 18,11 14 for unequal particle correlations and exploits the predicted properties of the transient, strange quark matter state [4,20,21, 19, even if it decays on strong interaction time scales. Using the description of this strangeness distillation process by a dynamical evolution model for the mixed QGP-hadronic phase, we have quantitatively demonstrated the sensitivity to the bag constant correlated to the stability of the quark matter possibly encountered in available experimental situations.

For the lower bag constant case a strong and sharp negative asymmetry effect is predicted in the correlation function ratio, thus offering a clear signal of the predicted strangeness distillation process provided the experimental resolution and statistics are sufficient to measure the ratio down to about $10 \mathrm{MeV} / \mathrm{c}$. Weaker though wider signals are predicted for the higher bag constant hypothesis. In this case the competition with other collision mechanisms generating the asymmetry is possible. These mechanisms, including the expansion of the hadronic system, are currently under investigation $[32,33]$.

Finally, we think that the unlike particle correlation technique may offer a very valuable tool to disentangle between different and presently debated scenarios for the phase transition in QCD matter at finite net baryon density.

\section{ACKNOWLEDGEMENTS}

The authors would like to thank B. Erazmus, L. Martin, D. Nouais, C. Roy, and A.Dumitru for useful discussions and/or for providing numerical programs. D. A. is pleased to thank the Institut für Theoretische Physik at the University of Frankfurt for invitation and kind hospitality. 


\section{REFERENCES}

[1] E. Witten, Phys. Rev. D30, 272 (1984).

[2] E. Farhi and R. .L. Jaffe, Phys. Rev. D38, 2379 (1984).

[3] H. Liu and G. Shaw, Phys. Rev. D30, 1137 (1984).

[4] C. Greiner, P. Koch, H. Stöcker, Phys. Rev. Lett. 58, 1825 (1987).

[5] C. Greiner, D. H. Rischke, H. Stöcker, P. Koch, Phys. Rev. D38, 2797 (1988).

[6] K. S. Lee and U. Heinz, Z. Phys. C43,425 (1989), U. Heinz, K. S. Lee, M. J. Rhoades-Brown, Mod. Phys. Lett. A2, 153 (1987).

[7] H. W. Barz, B. L. Friman, J. Knoll, H. Schulz, Nucl. Phys. A484, 661 (1988).

[8] R. Klingenberg (NA52 Collaboration), Proceedings of 12th International Conference on UltraRelativistic Nucleus-Nucleus Collisions (Quark Matter 96), Heidelberg (Germany), 1996. Nucl. Phys. A610, 306c (1996).

[9] F. S. Rotondo (E864 Collaboration), Proceedings of 12th International Conference on UltraRelativistic Nucleus-Nucleus Collisions (Quark Matter 96), Heidelberg (Germany), 1996. Nucl. Phys. A610, 297c (1996).

[10] M. J. Bennett (E878 Collaboration), Proceedings of 11th International Conference on UltraRelativistic Nucleus-Nucleus Collisions (Quark Matter 95), Monterey (California), 1995. Nucl. Phys. A590, 491c (1995).

[11] R. Lednicky, V. L. Lyuboshitz, B. Erazmus, D. Nouais, Rapport Interne 94-22 Nantes; submitted to Nucl. Phys. A.

[12] R. Lednicky, V. L. Lyuboshitz, B. Erazmus, D. Nouais, Phys. Lett. B373, 30 (1996). 
[13] B. Erazmus et al., ALICE Internal Note 95-43, Geneva, 1995.

[14] B. Erazmus, R. Lednicky, L. Martin, D. Nouais, J. Pluta, Proceedings of the XXXIV Bormio Meeting (1996).

[15] S. Soff et al., J. Phys. G, Nucl. Part. Phys. 23 (1997) 2095-2105. Special Issue of Int. Symposium on Strangeness in Quark Matter, Santorini, Greece (1997).

[16] M. Gyulassy, Phys. Lett. B286, 211 (1992).

[17] V.L.Lyuboshitz, M.I.Podgoretsky, Yad. Fiz. 30 (1979) 789 and Sov. J. Nucl. Phys. 30, 407 (1979).

[18] R. Lednicky, V. L. Lyuboshitz, Sov. J. Nucl. Phys. 35, 770 (1982); R. Lednicky, V. L. Lyuboshitz, Proceedings of the Int. Conf. on Nucl. Interferometry -CORINNE I- (1990) edited by D. Ardouin, World Scientific.

[19] C. Spieles, H. Stöcker, C. Greiner, Eur. Phys. J. C2, 351 (1998).

[20] C. Greiner, H. Stöcker, Phys. Rev. D44, 3517 (1991).

[21] C. Spieles, L. Gerland, H. Stöcker, C. Greiner, C. Kuhn, J. P. Coffin, Phys. Rev. Lett. 76, $1776(1996)$.

[22] For a review see e.g.: D. H. Boal, C. K. Gelbke, B. K. Jennings, Rev. Mod. Phy. 62553 (1990) and references therein.

[23] J. Pochodzalla et al., Phys. Rev. C35, 1695 (1987).

[24] J. Pochodzalla et al., Phys. Lett. B161, 256 (1985); J. Pochodzalla et al., Phys. Lett. B174, 36 (1986).

[25] B. Erazmus et al., Nucl. Phys. A583, 395 (1995); B. Erazmus, L. Martin, R. Lednicky, N. Carjan, Phys. Rev. C49, 349 (1994); B. Erazmus, N. Carjan, D. Ardouin, Phys. Rev. C44, 2663 (1991); J. M. Alexander, A. Elmaani, L. Kowalski, N. N. Ajitanand, C. J. Gelderloos, 
Phys. Rev. C48, 2874 (1993).

[26] L. Martin, C. K. Gelbke, B. Erazmus, R. Lednicky, Nucl. Phys. A604, 69 (1996).

[27] C. Gelderloos et al., Nucl. Inst. Meth. A349, 618 (1994).

[28] R. Lednicky, S. Panitkin, Nu Xu (in preparation); S. Voloshin, R. Lednicky, S. Panitkin, $\mathrm{Nu}$ Xu, Phys. Rev. Lett. 79, 4766 (1997).

[29] C. Ghisalberti et al., Proceedings of the XXXI Int. Bormio Meeting (1993), and Nucl. Phys. A583, 401 (1995).

[30] D. Gourio, PhD Thesis (1996), Nantes (unpublished).

[31] D. Nouais, PhD Thesis (1996), Nantes (unpublished).

[32] A. Dumitru et al., private communication and to be published.

[33] S. Soff et al., (1997) to be published 


\begin{tabular}{|r||r|r|r|r|}
\hline set & $\langle v t\rangle(\mathrm{fm})$ & $\left\langle r_{L}\right\rangle(\mathrm{fm})$ & $\left\langle r_{L}^{*}\right\rangle(\mathrm{fm})$ & $\left\langle r^{*}\right\rangle(\mathrm{fm})$ \\
\hline \hline 1 & -10.9 & 1.1 & 14.3 & 17.5 \\
\hline 2 & -1.4 & 0.5 & 2.6 & 5.5 \\
\hline 3 & -0.7 & 0.2 & 1.3 & 6.3 \\
\hline
\end{tabular}

TABLE I. Mean values of the relative space-time coordinates for pairs with relative momenta $q \leq 50 \mathrm{MeV} / \mathrm{c}$ calculated within the mixed phase thermodynamical model (see text). 
FIG. 1. Time evolution of baryon number $A$, strangeness fraction $f_{s}$, temperature $T$ and yields of $K^{+}$and $K^{-}$for set 1 (left column), set 2 (central column) and set 3 (right column) (see text).

FIG. 2. Ratios of correlation functions $\left(1+R^{+}\right) /\left(1+R^{-}\right)$for three parameter sets in a mixed-phase thermodynamical model (see text). The strange quark matter distillation process predicted in the case of a low bag constant (upper curve) results in a measurable deviation from unity in the range $q<30 \mathrm{MeV} / \mathrm{c}$. 


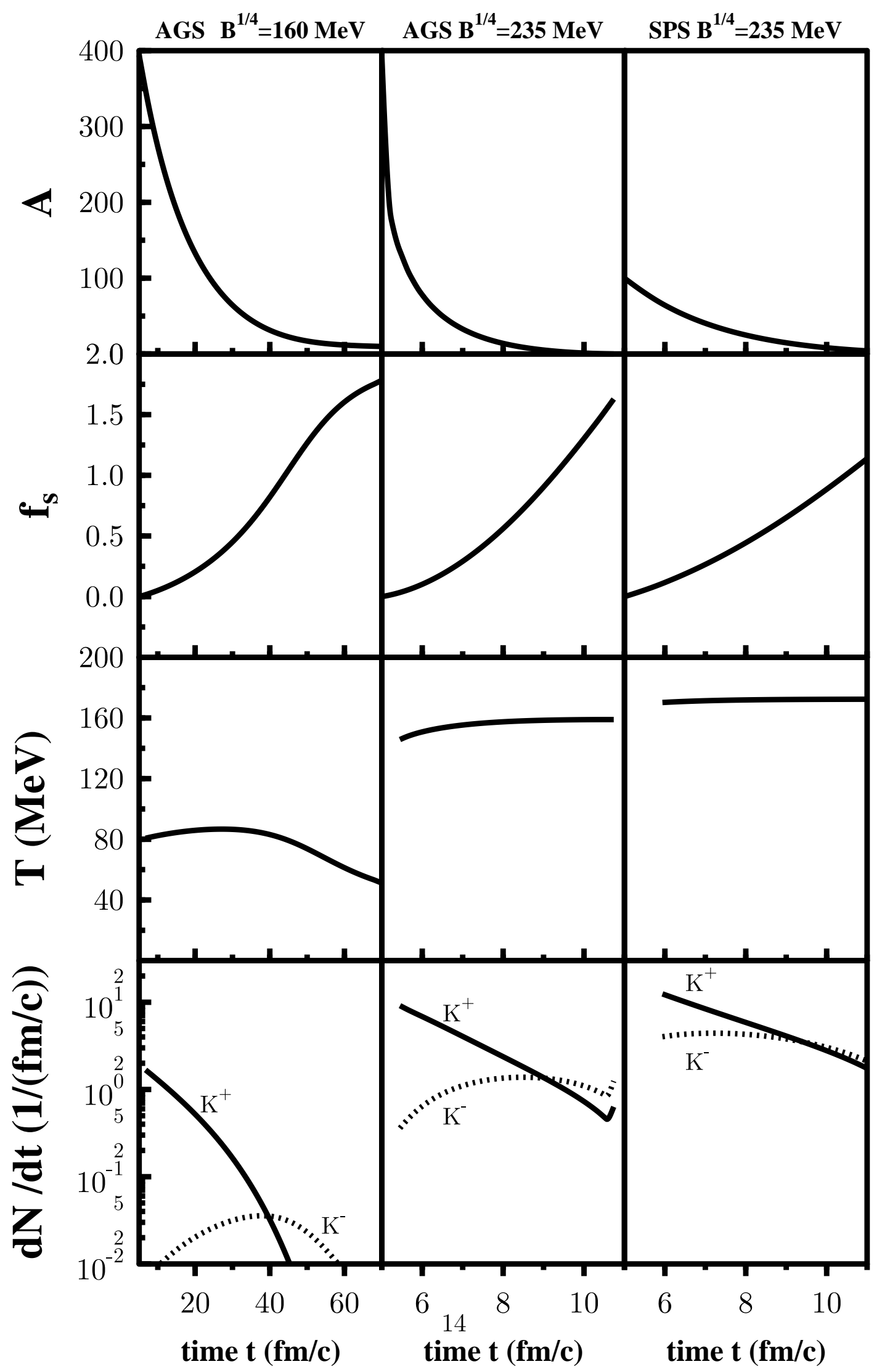




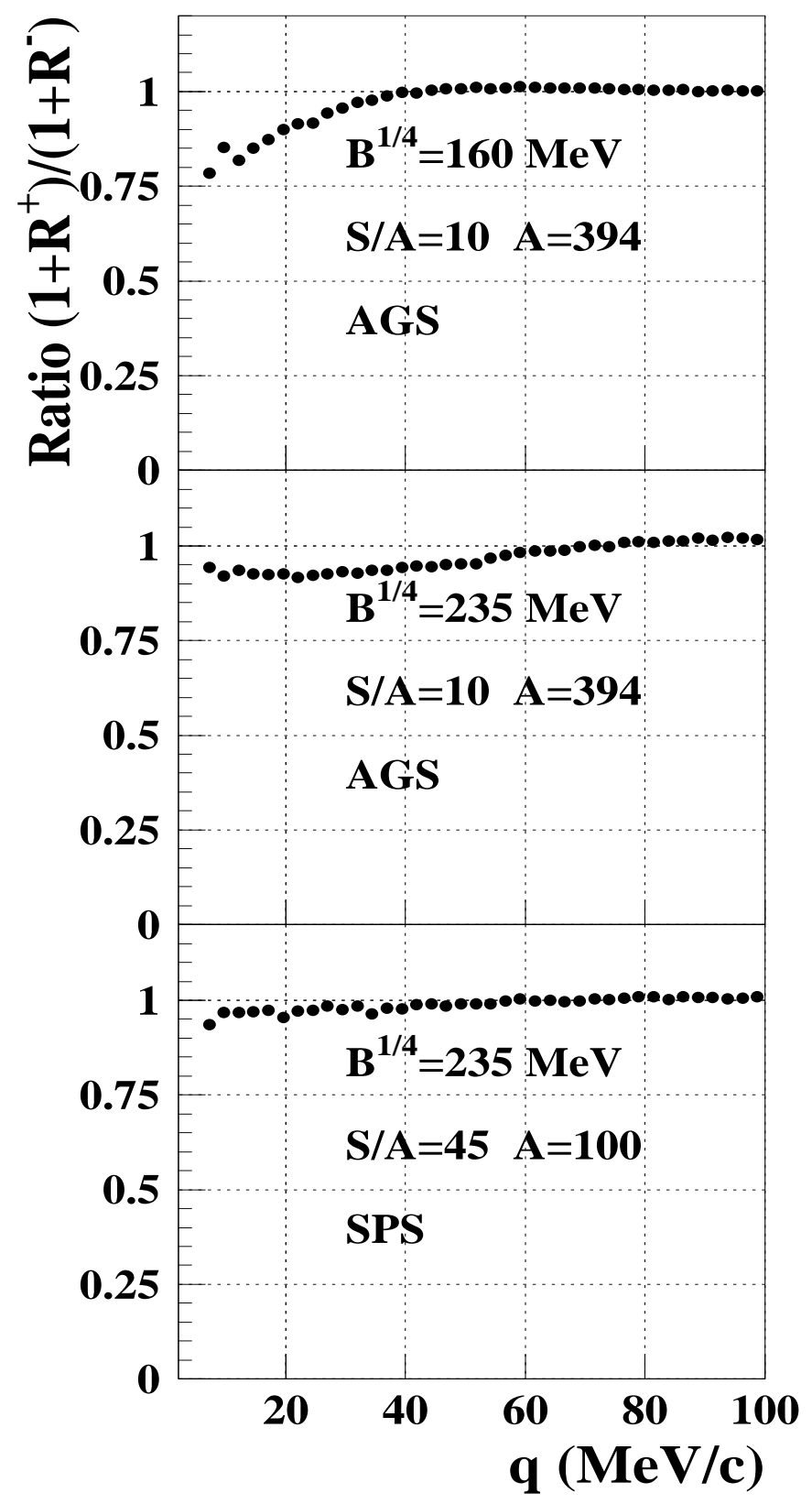

Fig.2 